\title{
Anodal Transcranial Direct Current Stimulation Temporarily Reverses Age-Associated Cognitive Decline and Functional Brain Activity Changes
}

\author{
Marcus Meinzer, ${ }^{1,2}$ Robert Lindenberg, ${ }^{1}$ Daria Antonenko, ${ }^{1}$ Tobias Flaisch, ${ }^{3}$ and Agnes Flöel ${ }^{1}$ \\ ${ }^{1}$ Department of Neurology, Charité University Medicine, 10117 Berlin, Germany, ${ }^{2}$ Center for Clinical Research, University of Queensland, Brisbane 4029, \\ Australia, and ${ }^{3}$ Department of Psychology, University of Konstanz, 78464 Konstanz, Germany
}

The rising proportion of elderly people worldwide will yield an increased incidence of age-associated cognitive impairments, imposing major burdens on societies. Consequently, growing interest emerged to evaluate new strategies to delay or counteract cognitive decline in aging. Here, we assessed immediate effects of anodal transcranial direct current stimulation (atDCS) on cognition and previously described detrimental changes in brain activity attributable to aging. Twenty healthy elderly adults were assessed in a crossover shamcontrolled design using functional magnetic resonance imaging (fMRI) and concurrent transcranial DCS administered to the left inferior frontal gyrus. Effects on performance and task-related brain activity were evaluated during overt semantic word generation, a task that is negatively affected by advanced age. Task-absent resting-state fMRI (RS-fMRI) assessed atDCS-induced changes at the network level independent of performance. Twenty matched younger adults served as controls. During sham stimulation, task-related fMRI demonstrated that enhanced bilateral prefrontal activity in older adults was associated with reduced performance. RS-fMRI revealed enhanced anterior and reduced posterior functional brain connectivity. atDCS significantly improved performance in older adults up to the level of younger controls; significantly reduced task-related hyperactivity in bilateral prefrontal cortices, the anterior cingulate gyrus, and the precuneus; and induced a more "youth-like" connectivity pattern during RS-fMRI. Our results provide converging evidence from behavioral analysis and two independent functional imaging paradigms that a single session of atDCS can temporarily reverse nonbeneficial effects of aging on cognition and brain activity and connectivity. These findings may translate into novel treatments to ameliorate cognitive decline in normal aging in the future.

\section{Introduction}

Because of the constant growth of the elderly population worldwide, the incidence of age-associated cognitive impairments and dementia will increase, imposing substantial burdens on societies (Grady, 2012). Therefore, the search for new strategies to maintain the integrity of higher brain functions in the elderly is of major importance. Transcranial direct current stimulation (tDCS), a noninvasive brain stimulation technique, may add to the repertoire of neuroscientists pursuing this challenge. During tDCS, weak electrical currents are applied to the skull to modulate neural activity. In particular, anodal tDCS (atDCS), which

Received Dec. 16, 2012; revised June 17, 2013; accepted June 20, 2013.

Author contributions: M.M. and A.F. designed research; M.M. and D.A. performed research; M.M., R.L., and T.F. analyzed data; M.M., R.L., and A.F. wrote the paper.

This work was supported by grants from the Deutsche Forschungsgemeinschaft (FI-379-8/1 and 379-10/1 to A.F. and DFG-Exc-257), the Bundesministerium für Bildung und Forschung (FKZ0315673A and 01GY1144 to A.F.; $01 E 00801$ to M.M.), the Else-Kröner Fresenius Stiftung (2009-141 to A.F.; 2011-119 to R.L.), and the Australian Research Council (ARC FT120100608 to M.M.). We thank Keren Avirame who helped us analyze response times.

The authors declare no competing financial interests.

Correspondence should be addressed to either Marcus Meinzer or Agnes Flöel, Department of Neurology, Center for Stroke Research Berlin, and Cluster of Excellence NeuroCure, Charité University Medicine, Charitéplatz 1, 10117 Berlin, Germany. E-mail:m.meinzer@uq.edu.au or agnes.floeel@charite.de.

DOI:10.1523/JNEUROSCI.5743-12.2013

Copyright $\odot 2013$ the authors $\quad 0270-6474 / 13 / 3312470-09 \$ 15.00 / 0$ reduces the threshold required for neuronal firing, can improve cognition and neural efficiency (Turi et al., 2012).

Surprisingly, studies assessing the impact of atDCS in older adults are scarce, but there is evidence that atDCS can improve cognition in healthy aging (Berryhill and Jones, 2012; Flöel et al., 2012). So far, only one study assessed neural correlates of atDCS in aging (Holland et al., 2011) and demonstrated improved word retrieval and enhanced neural priming compared with placebo stimulation ("sham"). However, priming is relatively unaffected by aging (Lustig and Buckner, 2004). Therefore, little is known about neural underpinnings of tDCS effects on tasks showing a decline across the life span. Moreover, to provide a comprehensive evaluation of atDCS on performance and brain functions in aging, it is necessary to assess the same parameters in younger adults, thereby providing evidence that the stimulation can reverse detrimental effects of aging.

The present study assessed immediate effects of atDCS on performance and brain functions in older adults using functional magnetic resonance imaging (fMRI) and simultaneous tDCS. A group of matched younger adults served as controls. Two independent fMRI paradigms addressed the impact of atDCS on brain functions in aging at the local and global levels: an overt semantic word generation assessed in parallel atDCS effects on performance and local brain activity. This task was specifically chosen because reduced performance in older compared with 


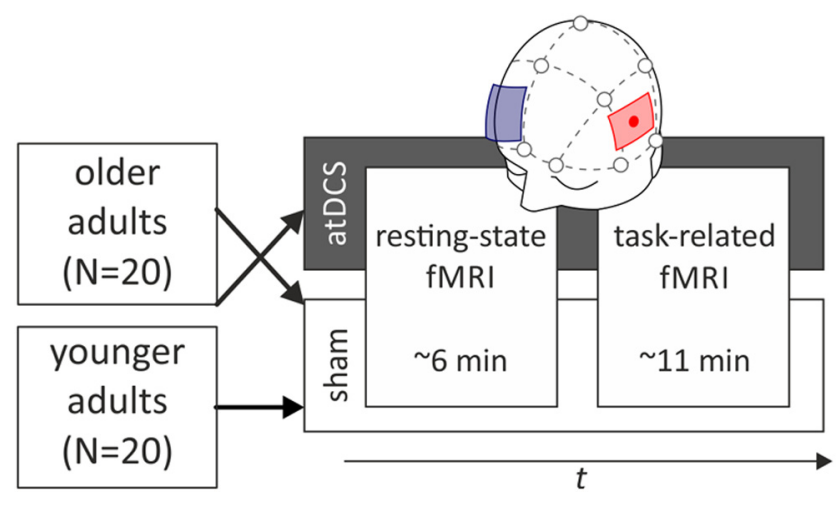

Figure 1. Design of the study. Twenty healthy older subjects participated in a crossover sham-controlled study. They were scanned during task-absent resting-state fMRI and overt semantic word generation (task-related fMRI) with concurrent tDCS (atDCS vs sham) administered to the left ventral inferior frontal gyrus (indicated by red rectangle; blue rectangle indicates position of reference electrode). Twenty matched healthy younger adults were also scanned using the same fMRI paradigms at the same scanner.

younger adults is typically accompanied by enhanced activity in bilateral prefrontal areas mediating different executive processes (Meinzer et al., 2009). In a crossover within-subject, shamcontrolled design, stimulation was administered to the left inferior frontal gyrus (IFG). The same tDCS montage improved word retrieval in younger adults and enhanced processing efficiency in left prefrontal cortex (Meinzer et al., 2012b). We hypothesized that atDCS would improve performance in older adults and reduce age-associated prefrontal hyperactivity.

In addition, resting-state fMRI (RS-fMRI) assessed the impact of atDCS at the network level independent of performance (Fox and Greicius, 2010). Specifically, enhanced connectivity of prefrontal areas has been linked to impaired language processing in older adults (Antonenko et al., 2012), and numerous studies demonstrated additional large-scale changes of RS network configuration in aging (for review, see Goh, 2011). Given that prefrontal atDCS exerted beneficial effects on bilateral languagerelated networks (Meinzer et al., 2012b) and the default mode and fronto-parietal attention networks (Keeser et al., 2011) in younger adults during RS-fMRI, we hypothesized that atDCS would induce a more "youth-like" connectivity pattern in prefrontal language-related regions but also in other networks negatively affected by advanced age (Goh, 2011).

\section{Materials and Methods}

Study outline. In a crossover within-subjects design, healthy elderly adults were assessed during two identical fMRI sessions (either with simultaneous atDCS or sham). The design of the present study was identical to a previous study that assessed the impact of tDCS on word-retrieval performance, task-related activity, and resting-state connectivity in younger adults (Meinzer et al., 2012b). To prevent carryover effects, sessions were separated by at least 1 week, and the order of stimulation was counterbalanced across the group. In both fMRI sessions, older adults were scanned during a resting-state sequence and a subsequent overt semantic word-generation task. To assess ageassociated changes in performance, brain activity, and connectivity, we compared data of the older adults with that of a matched younger control group (Fig. 1 illustrates the design of the present study). We used data of younger adults that were scanned using the same fMRI protocol and crossover designs comparing atDCS versus sham in previous studies of our work group (see below for details). For the present study, only data that was acquired during sham was used. The study was approved by the ethics committee of the Charité University Hospital and conducted in accordance with the Helsinki Declaration. Written informed consent was obtained from all participants before study inclusion.

Study rationale. The two fMRI paradigms (task related vs resting state) were included to address the impact of atDCS on two different phenomena that have both been associated with healthy aging. Task-related fMRI specifically addressed potential atDCS effects on (reduced) wordretrieval performance and previously described hyperactivity in bilateral prefrontal during language tasks in aging (for review, see Crosson et al., 2013). The RS-fMRI paradigm was included to provide additional evidence for atDCS effects at the functional network level. Please note that the stimulated left IFG is not only involved in language processing but also part of brain networks supporting different cognitive functions (e.g., working memory, attentional processing). Moreover, numerous studies provided evidence for age-associated changes in RS connectivity that have been linked to impairment of the latter functions as well (Goh, 2011; Ferreira and Busatto, 2013). Given that prefrontal atDCS resulted in modulations in a number of different functional networks in younger adults (Keeser et al., 2011; Meinzer et al., 2012b), the RS analysis aimed to explore beneficial effects of atDCS on network configuration on a much broader scale compared with the highly specific approach used for the task-related analysis (see below for details of our respective data analysis strategies).

However, despite the fact that the two fMRI paradigms addressed different phenomena, we aimed to address three common issues. First, we wanted to determine differences in performance and task-related activity (during semantic word generation) and resting-state connectivity between younger and older adults (both age groups in their "normal" state, i.e., during sham). Second, we wanted to assess the impact of atDCS on performance, task-related activity, and RS connectivity in the older group (i.e., the within-group comparison of atDCS vs sham). Third, we wanted to assess whether atDCS in the older group would result in a more youth-like pattern of behavioral performance, task-related activity, and RS connectivity (i.e., by directly comparing data of the younger control group acquired during sham with data of the older adults acquired during atDCS).

Participants. Twenty healthy older adults were recruited for this study (all native German speakers; 10 females and 10 males; age mean \pm SD, $68.0 \pm 5.7$ years; range, $60-76$ years). Each older subject completed a standard health questionnaire to exclude any previous or current neurological or psychiatric condition and the neuropsychological test battery of the Consortium to Establish a Registry for Alzheimer's Disease (CERAD-Plus; www.memoryclinic.ch), an established test to differentiate normal aging from dementia and its precursors. It comprises the Mini-Mental State Examination (MMSE; Folstein et al., 1975) and subtests assessing verbal fluency, naming, constructional praxis, and verbal memory. None of the older participants reported subjective memory complaints in everyday life or had a MMSE score below 27 (mean \pm SD, $28.9 \pm 0.9$ ). All participants scored within $1.5 \mathrm{SD}$ of the mean for the CERAD normative sample in all subtests, indicative of normal cognitive functioning. Ten older participants did not take any medication. Of the remaining 10 individuals, five substituted thyroxine, five took blood pressure-lowering medication, and three took statins.

As a control group, we used the data of 20 younger participants (age mean \pm SD, $26.4 \pm 3.4$ years; range, $19-31$ years; none reported use of psychoactive medication or recreational drugs) acquired during two previous studies of our work group that assessed the impact of atDCS versus sham on language ( $n=16$; Meinzer et al., 2012b) or language and motor functions ( $n=4$; Lindenberg et al., 2013) using crossover designs. Younger subjects were matched to the older subjects for sex (10 females, 10 males) and education (years of education mean \pm SD: young, $15.6 \pm$ 1.9; old, $15.9 \pm 1.2$ ) and were scanned at the same scanner using the identical fMRI setup. Only scans acquired during sham tDCS were used. To account for possible effects of repeated scanning, 10 of the younger subjects received sham tDCS during the first scanning session and the remaining 10 during the second session. All participants were strongly right hand according to the Edinburgh inventory (Oldfield, 1971) and participated for the first time in a tDCS study.

For all younger and older subjects, T1- and diffusion-weighted MRI images were acquired and inspected by a board-certified neuroradiolo- 
gist for any clinically relevant pathology (none found). In particular, there was no evidence of brain infarcts, including lacunar lesions, which are frequently associated with cognitive impairment in old age (Galluzzi et al., 2008). In older adults, an additional FLAIR (fluid-attenuated inversion recovery sequence) was acquired and rated for presence of white matter hyperintensities (WHM) according to the revised Fazekas Scale (Pantoni et al., 2010; List et al., 2011). No WMH was evident in eight participants (Fazekas score 0), and 12 older participants showed mild WMH (Fazekas score 1).

$t D C S$. The stimulation protocol was identical to that of a previous study (Meinzer et al., 2012b). A constant direct current (1 mA) was administered by an MRI-compatible stimulator (DC-Stimulator Plus; NeuroConn) during RS-fMRI and task-related fMRI. The stimulating electrode was inserted in a saline-soaked synthetic sponge $\left(5 \times 7 \mathrm{~cm}^{2}\right)$ and centered over the left ventral IFG (vIFG) using the 10-20 EEG system (Meinzer et al., 2012b). Electrode positions were verified on the T1-weighted images in every subject. A $10 \times 10 \mathrm{~cm}^{2}$ reference electrode was positioned over the right supraorbital region. The large size $(10 \times 10$ $\mathrm{cm}^{2}$ ) of the reference electrode renders the stimulation over the contralateral orbitofrontal cortex ineffective (Nitsche et al., 2007), and there is abundant behavioral evidence suggesting the specificity of this setup (for a recent review, see Nitsche and Paulus, 2011).

The current was turned on approximately $1 \mathrm{~min}$ before the resting-state scan and increased in a ramp-like fashion over $10 \mathrm{~s}$, eliciting a tingling sensation on the scalp that fades over seconds. The current was turned off after $30 \mathrm{~s}$ (sham) or continued for a total of $20 \mathrm{~min}$ in all subjects during atDCS (i.e., active stimulation outlasted the task by $\sim 20-120$ s). The study was conducted in a double-blind fashion (i.e., the subject and the researcher who conducted the MRI scans, interacted with the subjects and conducted the behavioral assessments, were blind to the stimulation condition). A separate investigator administered the stimulation. Two self-report rating scales were administered immediately before and after the two scanning sessions to assess mood and positive and negative effect [Visual Analog Mood Scales (VAMS) (Folstein and Luria, 1973); Positive and Negative Affect Scales (PANAS) (Watson et al., 1988)].

fMRI setup and acquisition parameters. Scanning was conducted using a 3 tesla Siemens Trio MR System at the Berlin Center for Advanced Neuroimaging. Initially, a continuous RS-fMRI sequence was acquired $\left(3 \times 3 \times 4 \mathrm{~mm}^{3}\right.$; TR/TA, $2300 \mathrm{~ms}$; TE, 30 ; flip angle, $90^{\circ}$; 34 transverse slices; no gap; interleaved acquisition; FOV, $192 \times 192$; acquisition matrix, $64 \times 64 ; 150$ functional volumes). Subjects were instructed to keep their eyes closed, relax, think of nothing particular, and move as little as possible. A T2*-sensitive echoplanar imaging BOLD sequence was acquired to assess task-related functional activity during overt semantic word retrieval. Details of the task and stimulus selection have been described previously in detail (Meinzer et al., 2012b). In short, the paradigm used a temporal sparse-sampling design, in which the overt verbal response was assessed during a scanner off phase, and the hemodynamic response was acquired after a short time delay to avoid movement artifacts during articulation $\left(3 \times 3 \times 3 \mathrm{~mm}^{2}\right.$; TR, 6000; TA, 2000; TE, 30; flip angle, $90^{\circ}$; 32 transverse slices; gap, $0.75 \mathrm{~mm}$; slice thickness, $3 \mathrm{~mm}$; interleaved acquisition; FOV, $192 \times 192$; acquisition matrix, $64 \times 64$; 104 functional whole-brain images). During each scanning session, participants were visually presented with six semantic categories ( 10 consecutive trials, $3.8 \mathrm{~s}$ per trial; i.e., total of 60 word-generation trials) and asked to overtly generate different exemplars for each category. After each trial, the stimulus disappeared and was replaced by a black screen $(2.2 \mathrm{~s})$, and a whole-brain functional volume was acquired. Task blocks alternated with a baseline condition (five consecutive trials of saying the word "rest"). Two different sets of categories (matched for linguistic criteria) were used during the two fMRI sessions and counterbalanced across subjects (for details, see Meinzer et al., 2012a). Verbal responses were recorded and subsequently transcribed. Responses were scored by two independent raters blinded to the stimulation condition. In case of disagreement, a consensus was reached. Incorrect responses (exemplars that did not belong to a given category), omissions, and repetitions (e.g., same response or synonyms) were scored as errors. Consecutive answers that started with the same letter were not scored as errors (except if they were repetitions or synonyms).
Task-related fMRI data analysis. Statistical Parametric Mapping (SPM5; Wellcome Department of Imaging Neuroscience, London, UK) was used for task-related fMRI analysis. Preprocessing comprised functional image realignment, coregistration with the individual participants' anatomical images, unified segmentation and registration to MNI standard space, and spatial smoothing with an $8 \times 8 \times 8 \mathrm{~mm}$ Gaussian kernel. The design matrix for the statistical analysis comprised the covariates of interest (semantic word-generation and baseline trials) and movement parameters. Only correct trials were included in the analysis; incorrect trials were included as additional covariates of no interest in the statistical model. Before model estimation, a high-pass filter (128s) was applied and data were modeled with a finite impulse response (Meinzer et al., 2012b). Planned contrasts of interest were estimated on the individual subject level for each session (semantic word-generation vs baseline trials) in both age groups. A random-effects whole-brain comparison of correct semantic word-generation trials with the baseline condition that comprised data of younger and older adults during sham (i.e., the main effect of task; $n=40$ ) was calculated to assure that (1) the task elicited a similar pattern of activity as in our previous studies (Meinzer et al., 2009, 2012c) and (2) brain areas chosen for a subsequent a priori region-of-interest (ROI) analysis were located in areas active during the task.

This ROI analysis focused on bilateral prefrontal brain regions where activity differences between younger and older subjects are most pronounced (Park and Reuter-Lorenz, 2009). Left vIFG was selected because it has been implicated specifically with semantic retrieval processes (Thompson-Schill et al., 1997) and showed decreased activity in our previous study in younger adults during atDCS compared with sham (Meinzer et al., 2012b). Left dorsal IFG (dIFG), implicated with more general selection or phonological retrieval processes, was chosen to assess the specificity of the stimulation [i.e., in an area that was located in the vicinity of the active electrode but was not expected to show task-related activity changes because of the stimulation (Meinzer et al., 2012b)]. Right vIFG and middle frontal gyri (MFG) assessed potential remote effects of the stimulation on activity in right frontal areas in the older adults. Both areas have frequently been shown to exhibit increased activity in older compared with younger subjects during language (Meinzer et al., 2009, 2012c; Goh, 2011) and nonlanguage (Park and Reuter-Lorenz, 2009) tasks possibly caused by enhanced demands placed on executive control processes in aging (Spreng et al., 2010).

For the ROI analysis, $6 \mathrm{~mm}$ spherical ROIs were created centered around Talairach coordinates -48/23/2 (left vIFG; BA 45/47) and -42/ 30/23 (left dIFG; BA 46/9) and the contralateral homolog of the left vIFG (Meinzer et al., 2012b). The right MFG (Talairach coordinate 33/42/17) was selected based on a previous study that used the same task (contrast, semantic fluency $>$ rest) in an independent sample of older native German speakers (Meinzer et al., 2009). To account for individual variability in anatomy and functional activity, mean beta activity was extracted from spherical ROIs $(6 \mathrm{~mm})$ centered around the individual participants' peak voxel from the contrast "word generation $>$ baseline" in the abovedescribed ROIs for each session in younger (sham) and older subjects (atDCS; sham). All ROIs were located within areas activated by younger and older subjects during the word-generation task (i.e., the main effect of task). We conducted repeated-measures ANOVAs that assessed activity differences in the four a priori ROIs between younger and older adults and comprised the within-subjects factor REGION and the betweensubjects factor AGE GROUP. This analysis was conducted separately for the comparison of the younger subjects' data (acquired during sham) with that of the older subjects acquired during sham and atDCS. An additional analysis assessed the impact of atDCS compared with sham in the older group and comprised the within-factors REGION and STIMULATION. We report Greenhouse-Geisser corrected results of significant effects of interest. Post hoc analyses comprised paired or unpaired $t$ tests as appropriate. Statistical analyses were performed using SPSS (version 21; IBM). Associations between activity (during sham tDCS) or activity changes (sham vs anodal tDCS) in the four ROIs in the older group with performance or tDCS-induced performance improvements, respectively, were explored using Pearson correlation coefficients. 
To gain additional information about the distribution of stimulation effects in the older adults, we also conducted an exploratory whole-brain analysis that directly compared data acquired during atDCS and sham using a paired $t$ test as implemented in SPM. We report results of significant voxels within significant clusters (voxel level, $p<0.001$ uncorrected; cluster level, $p<0.05$ family-wise error corrected).

$R S$-fMRI data analysis. For RS-fMRI data analysis, Eigenvector Centrality Mapping (ECM; Lohmann et al., 2001) was chosen. ECM is an established graph-based approach that can be used to quantitatively characterize complex network structures across the entire brain without requiring a priori assumptions about the underlying network structure. A major feature of ECM is its inherently explorative nature (Zuo et al., 2012). Unlike other types of functional connectivity measures (e.g., seedbased approaches), ECM does not require any a priori assumptions about the underlying network structure while taking into account the interconnectedness of central brain regions ("hubs") across the entire functional connectome (Zuo et al., 2012). Given that the exact impact and extent of atDCS-induced electrical fields is currently unknown (Keeser et al., 2011), and that the impact of atDCS on resting-state functional connectivity in aging had not been studied so far, the abovedescribed features rendered ECM as an ideal tool for the present investigation. Moreover, specificity of networks changes with advanced age, i.e., networks that are relatively independent from each other in younger adults may show stronger interconnectedness in older adults (Geerligs et al., 2012), and a number of different large-scale networks, including the default, salience, motor, and visual networks, can be affected by advanced age and show enhanced or reduced connectivity (Ferreira and Busatto, 2013). Therefore, focusing on a single network (i.e., using a seed-based approach) would potentially underestimate the impact of atDCS on the aging brain. In contrast, by using the ECM it was possible to assess the impact of tDCS not only on one specific network (or a number of arbitrary selected networks) but, rather, capture complex changes induced by atDCS.

Preprocessing of RS-fMRI data was performed using LIPSIA (Leipzig Image Processing and Statistical Inference Algorithms) and ECM (Lohmann et al., 2001, 2010). During ECM, a centrality value is attributed to each brain voxel, with higher values indicating voxels that are more strongly connected to other voxels central within networks (Lohmann et al., 2010). As in previous studies, of our work group (Meinzer et al., 2012b; Lindenberg et al., 2013) we applied spectral ECM of lowfrequency bands $(<0.08 \mathrm{~Hz})$. This approach was first suggested by Lohmann et al. (2010). It allows for including only well described frequencies that have been found to be valuable for assessing interregional dependencies (Salvador et al., 2005) and to inspect individual frequency bands for physiological noise before averaging (Lohmann et al., 2010). Preprocessing included motion and slice time correction, spatial normalization to the LIPSIA template, bandpass filtering at $1 / 90 \mathrm{~s}$, and spatial smoothing with a $6 \times 6 \times 6 \mathrm{~mm}$ Gaussian kernel. The analysis was restricted to the gray matter using a binary mask obtained from a study-specific template created with FSL after segmenting the anatomical T1 images of all subjects (FSL-VBM, http://www.fmrib.ox.ac.uk/fsl). One older participant was excluded from this analysis because of rhythmic leg movements and head movements $(>8 \mathrm{~mm})$ during the RS-fMRI scan. Individual voxel-wise spectral coherence analysis was conducted for eight frequency intervals $(0.01-0.08 \mathrm{~Hz})$ for the atDCS and sham conditions separately (sham tDCS only in the younger group). After $z$-transformation of the resulting matrices (Lohmann et al., 2010), the respective spectral bands were averaged on an individual basis, and the resulting mean images of younger (during sham) and older (atDCS and sham) participants were compared by whole-brain unpaired or paired $t$ tests. Clusters were considered significant at $p<.05$, corrected for multiple comparisons using the Monte Carlo simulation (Lohmann et al., 2010).

\section{Results}

Impact of atDCS on behavioral performance

Similar to previous studies that compared performance during semantic word retrieval in healthy younger and older adults (Meinzer et al., 2009, 2012a,c), older participants produced sig-

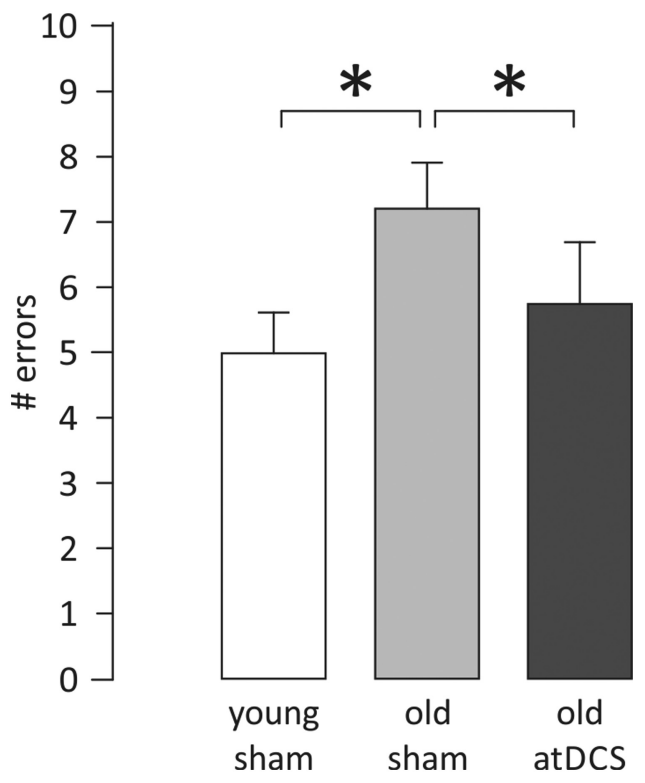

Figure 2. Performance accuracy during the semantic word-generation task in the two age groups. During sham, older adults produced significantly fewer correct exemplars during the task compared with younger adults (i.e., more errors). During atDCS, performance in older adults improved significantly up to the level of the matched younger control group. Data show mean \pm SEM; $n=20$ per age group. ${ }^{*} p<0.05$.

nificantly more errors compared with the matched younger control group during sham $\left(t_{(38)}=2.43, p=0.019\right)$. Moreover, in line with previous studies demonstrating improved word retrieval attributable to atDCS administered to left prefrontal areas (Iyer et al., 2005; Fertonani et al., 2010; Cattaneo et al., 2011; Meinzer et al., 2012b), older participants produced significantly fewer errors during atDCS compared with sham participants $\left(t_{(19)}=-2.63, p=0.016\right)$. During atDCS, performance of the older group improved up to the level of the younger group, as indicated by a nonsignificant difference between participants of the two age groups $\left(t_{(38)}=0.46, p=.50\right.$; see Fig. 2 for details). Response times were comparable between younger and older adults during sham stimulation (mean \pm SD; young, $859 \pm 246$; old, $872 \pm 169 ; t_{(39)}=91, p=0.91$ ), and no differences in response times were found between atDCS and sham stimulation in the older group (mean \pm SD; sham, $872 \pm 169$; atDCS, $857 \pm$ $\left.127 ; t_{(19)}=0.80, p=0.43\right)$.

\section{Impact of atDCS on task-related activity}

The random-effects whole-brain comparison of correct semantic word-generation trials with the baseline condition that comprised data of younger and older adults during sham confirmed a similar pattern of activity as reported in previous studies that used the same paradigm (Meinzer et al., 2009, 2012c). Activity was most pronounced in the left dIFG and bilaterally in the vIFG and insula, MFG, caudate nuclei, and medial frontal areas. Taskrelated activity for this contrast is illustrated in Figure $3 \mathrm{~A}$.

\section{Impact of atDCS on activity in a priori ROIs}

Activity differences between the two age groups (during sham) in the four a priori ROIs and the effect of stimulation in the older group (sham vs atDCS) are illustrated in Figure 3B. Repeatedmeasures ANOVA results revealed significant main effects of AGE GROUP for the comparison of younger versus older adults during sham $\left(F_{(1,38)}=15.32, p<0.0001\right)$. Post hoc tests showed that older adults exhibited more pronounced activity than the 
A

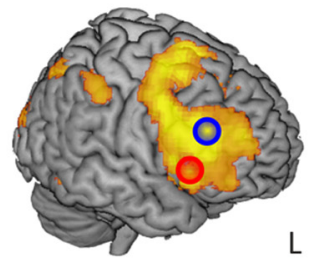

$\mathrm{L}$

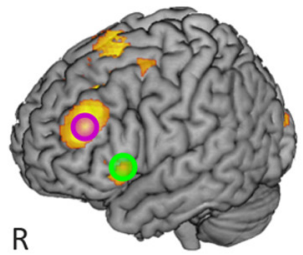

B

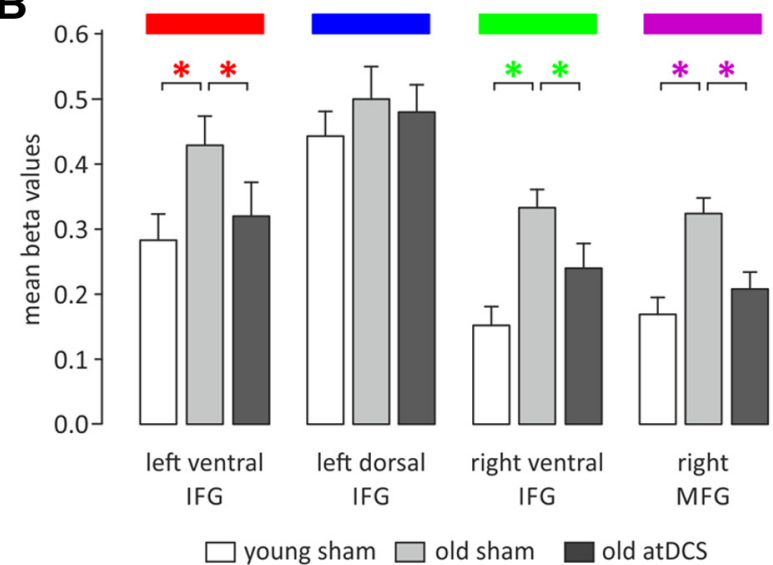

C
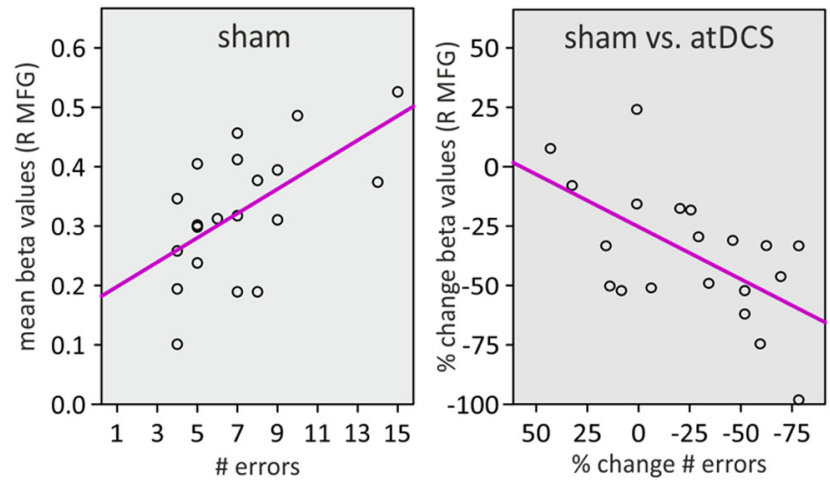

Figure 3. Task-related fMRI analysis. $\boldsymbol{A}$, The main effect of task (correct semantic wordgeneration trials vs baseline; $p<0.01$, family-wise error corrected for multiple comparisons, cluster extent $k=20$ ) and location of the a priori ROls in bilateral prefrontal areas: left ( $L$; red) and right (R; green) ventral IFG; left dorsal IFG (blue); right MFG (purple). $B$, Effect of atDCS (compared with sham) on activity in a priori ROls. atDCS resulted in significantly reduced activity in bilateral ventral IFG and right MFG that was hyperactive in the older group during sham; left dorsal IFG was not hyperactive on the older group and was unaffected by the stimulation. ${ }^{*} p<0.05$. C, Correlation analysis. Enhanced activity in right MFG was associated with reduced performance in older adults (during sham); improved performance during atDCS (i.e., percentage of reduction of errors during word generation) was associated with reduced activity in right MFG. Data show mean \pm SEM.

younger participants in the ventral portion of the IFG $\left(t_{(38)}=\right.$ $2.45, p=0.018)$. Moreover, in line with previous studies that compared activity patterns during the same task (Meinzer et al., 2009, 2012c), older participants exhibited more pronounced activity in the right vIFG and the right MFG compared with the younger group during sham tDCS (right vIFG/MFG: $t_{(38)}=4.56 /$ 4.40 , both $p<0.0001)$. Differences between younger and older adults in the right IFG and MFG survived a Bonferroni's corrected threshold ( $p<0.0125$ ); the effect in the left vIFG was marginally significant. No significant differences were found between younger and older adults in the left $\operatorname{dIFG}\left(t_{(38)}=0.84, p=0.36\right)$.

The repeated-measures ANOVA that assessed tDCS-induced changes in the older group revealed a significant main effects of STIMULATION $\left(F_{(1,19)}=14.23, p=0.001\right)$. Post hoc paired $t$ tests confirmed that activity in the left vIFG decreased significantly in the older group during atDCS $\left(t_{(19)}=-2.94, p=\right.$ 0.008 ), and activity in both right frontal ROIs in the older group decreased significantly (right vIFG/MFG: $t_{(19)}=-2.16 /-4.92$, $p=0.043 / 0.001)$. Except for the right vIFG, activity reductions during atDCS survived Bonferroni's correction. As in our previous study (Meinzer et al., 2012a), atDCS did not affect activity levels in the dIFG compared with sham in the older group $\left(t_{(19)}=\right.$ $-0.50, p=0.62$ ). During atDCS, activity levels in all a priori ROIs were comparable between younger and older adults (nonsignificant effect of AGE GROUP: $F_{(1,19)}=1.38, p=0.198$; post hoc tests: left vIFG, $t_{(38)}=0.56, p=0.57$; left dIFG, $t_{(38)}=$ $0.65, p=0.52$; right IFG, $t_{(38)}=1.83, p=0.07$; right $\mathrm{MFG}$, $\left.t_{(38)}=1.07, p=0.29\right)$.

In addition, to explore potential stimulation effects on baseline activity, we also extracted mean beta activity in the four a priori ROIs for the baseline trials (i.e., compared with the "implicit baseline" implemented in SPM. The same repeatedmeasures ANOVA as above revealed no significant effect of the factor STIMULATION $\left(F_{(1,19)}=0.40 ; p=0.54\right.$; mean \pm SD beta activity: atDCS/sham left VIFG, $-0.10 \pm 0.20 /-0.08 \pm 0.19$; left $\mathrm{dIFG},-0.12 \pm 0.12 /-0.11 \pm 0.12$; right vIFG, $-0.10 \pm 0.14 /$ $-0.08 \pm 0.16$; right MFG, $-0.05 \pm 0.10 /-0.09 \pm 0.08$; all post hoc paired $t$ tests, $p>0.22$ ). Therefore, our findings cannot be explained by a selective effect of the stimulation on baseline trials. However, future studies are indicated to address a possible impact of atDCS on baseline glucose metabolism.

A correlation analysis revealed that enhanced activity in the right MFG was associated with poorer performance in the older group during sham tDCS $(r=-0.59, p=0.007)$. Moreover, decreased activity during atDCS was positively correlated with improved performance relative to sham tDCS $(r=0.58, p=$ 0.006; Fig. 3C). No additional correlations were observed between performance and activity during sham tDCS and tDCSinduced changes in performance and activity. Please note that removing linear trends of age by partial correlation analysis revealed that age did not affect these significant results (agecorrected correlations: activity sham vs performance sham, $p=$ 0.007; change activity vs change behavior, $p=0.009)$.

\section{Exploratory whole-brain analysis of tDCS effects on task-related activity}

A whole-brain paired $t$ test revealed no significant activity increases in the older group during active stimulation (i.e., the contrast atDCS $>$ sham). The reverse contrast ( sham $>$ atDCS) revealed two significant clusters outside of the lateral prefrontal lobe, indicative of reduced activity during atDCS compared with sham. These clusters were located in the anterior cingulate gyrus (BA $32,0 / 44 / 6, k=76, Z=3.9$ ) and the left precuneus (BA 7, $-12 /-69 / 36, k=46, Z=3.7)$. To explore whether these areas would show a similar pattern as our a priori ROIs (i.e., enhanced activity compared with younger adults during sham and comparable activity during atDCS), we extracted mean beta activity from ROIs centered around the peak voxels from the above whole-brain analysis in both areas in younger and older adults. During sham, older adults showed more pronounced activity in both areas than younger adults [anterior cingulate gyrus: mean \pm SD young/old (sham): $-0.03 \pm 0.18 / 0.18 \pm 0.17, t_{(38)}=3.9, p=$ 0.0003 ; precuneus: $0.02 \pm 0.12 / 0.18 \pm 0.20, t_{(38)}=3.0, p=$ $0.004]$. Activity decreased significantly in the older group in both ROIs during atDCS compared with sham [anterior cingulate gyrus mean \pm SD old (atDCS): $0.08 \pm 0.17, t_{(19)}=2.6, p=0.016$ precuneus: $\left.0.02 \pm 0.19, t_{(19)}=3.55, p=0.002\right)$, and activity in 

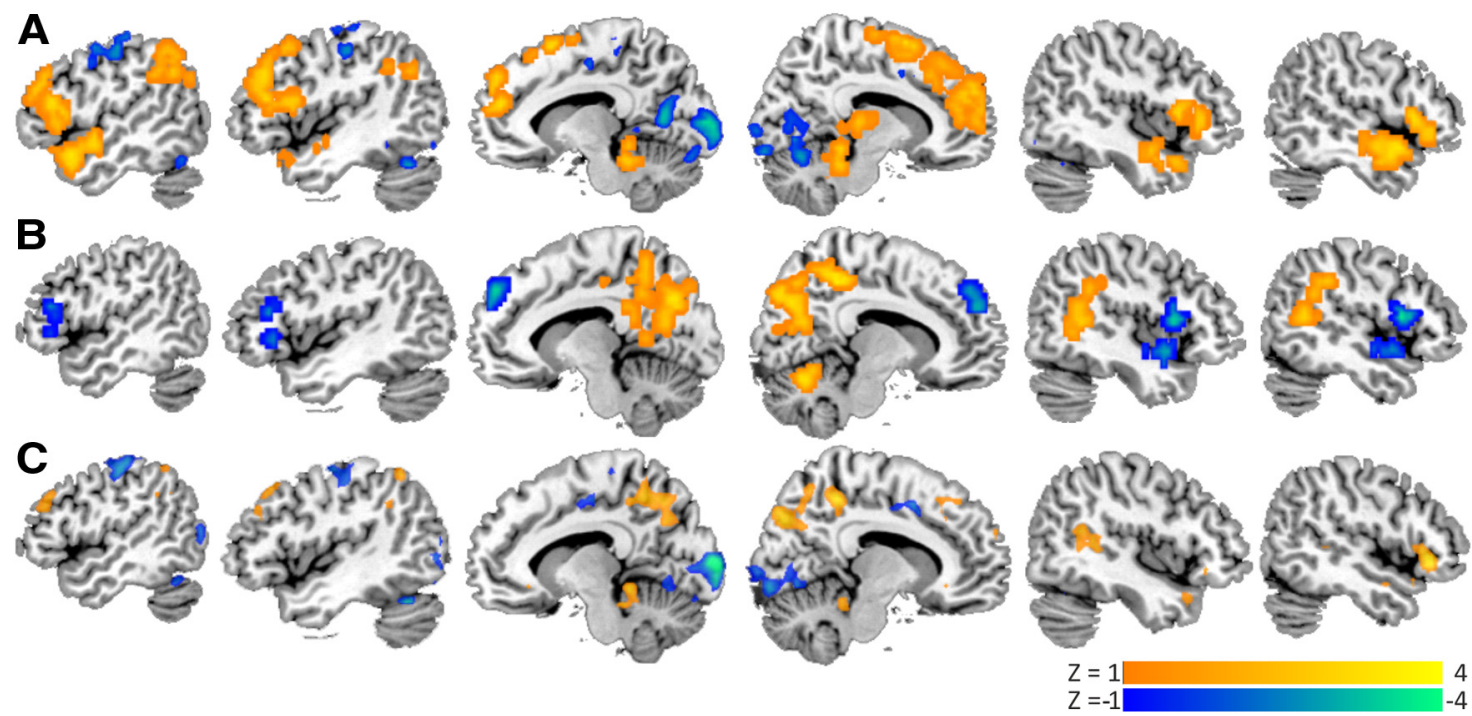

Figure 4. Resting-state analysis. A, Areas that exhibited enhanced (orange) or reduced (blue) connectivity in the older $(n=19)$ compared with the younger ( $n=20)$ group during sham tDCS. $\boldsymbol{B}$, Areas that exhibited enhanced (orange) or reduced connectivity (blue) during atDCS compared with sham in the older group. $\boldsymbol{C}$, Areas that exhibited enhanced (orange) or reduced (blue) connectivity in the older adults during atDCS compared with the younger group. Images on the left/right correspond with the respective hemispheres. Clusters were significant at $p<0.05$, corrected for multiple comparisons using Monte Carlo simulation. Coordinates of sagittal slices were as follows: $x=49 / 45 / 10$ (right hemisphere), $x=-49 /-45 /-10$ (left hemisphere).

the precuneus was now comparable in the two age groups $\left(t_{(38)}=\right.$ $0.01, p=0.94)$. Despite significantly reduced activity in the anterior cingulate gyrus when comparing atDCS with sham, activity was still higher in older adults during atDCS than in the younger group $\left(t_{(38)}=2.2, p=0.034\right)$. Activity changes were not correlated with behavioral improvement (anterior cingulate: $r=0.12$, $p=0.61$; precuneus: $r=-0.26, p=0.26$ ).

\section{Impact of atDCS on functional network connectivity during RS-fMRI}

ECM analysis revealed distinct differences in functional connectivity patterns in the two age groups during sham tDCS. Compared with the younger group, older subjects showed increased connectivity (i.e., referred to as "hyperconnectivity" from here on) in several anterior brain regions that was most pronounced in bilateral fronto-temporal and medial frontal brain regions. In contrast, older participants showed reduced connectivity (i.e., "hypoconnectivity") mainly in posterior brain regions, including temporo-occipital and precentral and postcentral cortices (see Fig. $4 A$ and Table 1 for details).

This pattern was partially reversed during atDCS in the older group (Fig. 4B, Table 2). In particular, bilateral fronto-temporal cortices (including areas that were hyperconnected during sham like bilateral IFG) exhibited a decrease in connectivity. A different pattern became evident for posterior brain regions. Here, a number of posterior midline areas, including bilateral posterior cingulate gyrus and precuneus (i.e., areas overlapping with the default mode network; Andrews-Hanna et al., 2007), exhibited increased connectivity during atDCS. In addition, increased connectivity in the posterior cingulate gyrus during atDCS overlapped with areas that were hypoconnected during sham in older compared with younger adults. However, ECM analysis also revealed that not all age-associated changes in connectivity were reversed by the stimulation, as demonstrated by the direct comparison of younger adults (sham) and older adults during atDCS (for details, see Table 2 and Fig. 4C). Therefore, atDCS resulted only in partial reversal of age-associated changes in connectivity. We also explored whether changes
Table 1. Differences in connectivity between younger and older adults during sham stimulation

\begin{tabular}{llll}
\hline Anatomical location $(\mathrm{BA})$ & $k$ & $\begin{array}{l}\text { Maximum } \\
Z \text { value }\end{array}$ Peak voxel $(x / y / z)$ \\
\hline
\end{tabular}

Older adults (sham) $>$ younger adults

Left insula, BA 13

Left inferior/middle frontal gyrus, BA 45

Left hippocampus

Right superior temporal gyrus, BA 22

Right hippocampus

Right superior frontal gyrus, BA 8

Right/left medial frontal gyrus, BA 6

Right insula, BA 13

Right inferior frontal gyrus, BAs 45/47

Left superior temporal gyrus, BA 38

Left/right superior frontal gyrus, BA 6

Left/right cerebellum

Left supramarginal gyrus, BA 40

Older adults (sham) $<$ younger adults

Left/right lingual gyrus, BA 18

Left fusiform gyrus, BA 37

Left superior parietal lobe, BA 7

Left postcentral gyrus, BA 3

Left paracentral lobe, BA 6

Left/right medial frontal gyrus, BA 6

Left/right cingulate gyrus, BA 24

Left/right cingulate gyrus, BA 31

\begin{tabular}{rcc}
537 & 5.7 & $-32 / 18 / 15$ \\
66 & 4.5 & $-32 /-9 /-16$ \\
& & \\
464 & 4.2 & $52 / 6 /-7$ \\
339 & 4.1 & $9 / 30 / 45$ \\
106 & 4.0 & $14 / 6 / 56$ \\
& & \\
200 & 3.8 & $39 / 10 / 1$ \\
85 & 3.7 & $-52 / 10 /-18$ \\
71 & 3.4 & $-12 / 12 / 64$ \\
70 & 3.3 & $-3 /-43 /-14$ \\
77 & 3.0 & $-49 /-57 / 34$ \\
& & \\
1135 & -4.6 & $-3 /-75 /-2$ \\
93 & -3.4 & $-38 /-57 /-16$ \\
55 & -4.2 & $-29 /-54 / 64$ \\
292 & -4.0 & $-23 /-27 / 67$ \\
114 & -3.9 & $-3 /-33 / 72$ \\
& & \\
180 & -3.7 & $-2 /-13 / 49$ \\
60 & -3.0 & $-3 /-48 / 26$ \\
\hline
\end{tabular}

$B A$, Brodman area; $k$, cluster extent (number of voxels); $x / y / z$, coordinates of peak voxel in significant clusters (Talairach space); if clusters extend into both hemispheres, this is indicated by left/right (if peak voxel is located in the left hemisphere) or right/left (if peak voxel is located in the right hemisphere).

found in the ECM analysis would overlap with our a priori ROIs chosen for the task-related analysis and the two areas identified in the additional whole-brain analysis. Except for two areas (left dorsal IFG and precuneus), there was no overlap between these areas. Changes in task-related activity and ECM values were not correlated (dIFG, $p>0.25$; precuneus, $p>0.93$ ), which highlights the independent contribution of the two fMRI paradigms to study atDCS effects on brain functions in aging. 


\begin{tabular}{|c|c|c|c|}
\hline Anatomical location (BA) & k & $\begin{array}{l}\text { Maximum } \\
\text { Z value }\end{array}$ & $\begin{array}{l}\text { Peak voxel } \\
(x / y / z)\end{array}$ \\
\hline \multicolumn{3}{|c|}{ Older adults: increased connectivity (atDCS > sham) } & \\
\hline Left/right precuneus, BA 7 & 1058 & 4.8 & $0 /-36 / 42$ \\
\hline Right superior temporal gyrus, BA 39 & 120 & 4.3 & $52 /-52 / 13$ \\
\hline \multicolumn{4}{|c|}{ Older adults: decreased connectivity (atDCS < sham) } \\
\hline $\begin{array}{l}\text { Left middle occipital gyrus, BA } 19 \\
\text { Right insula, BA } 13 \\
\text { Right inferior frontal gyrus, BA } 44\end{array}$ & 26 & -4.9 & $\begin{array}{l}-29 /-84 / 12 \\
40 / 10 / 4 \\
48 / 15 / 10\end{array}$ \\
\hline Right superior temporal gyrus, BA 38 & 116 & -4.5 & $48 / 5 /-12$ \\
\hline $\begin{array}{l}\text { Right/left medial frontal gyrus, BA } 8 \\
\text { Left middle frontal gyrus, BA } 46 \\
\text { Left inferior frontal gyrus, BA } 45\end{array}$ & 155 & -3.8 & $6 / 45 / 42$ \\
\hline Left insula, BA 13 & 102 & -3.6 & $-40 / 24 / 23$ \\
\hline \multicolumn{4}{|l|}{ Older adults (anodal) $>$ younger adults } \\
\hline Left middle frontal gyrus, BA 8 & 155 & 4.3 & $-38 / 27 / 48$ \\
\hline Right/left precuneus, BA 7 & 663 & 4.1 & $9 /-45 / 45$ \\
\hline Right inferior frontal gyrus, BA 45 & 116 & 4.0 & $46 / 27 / 1$ \\
\hline Left/right cerebellum & 225 & 4.0 & $-6 /-42 /-7$ \\
\hline Right superior temporal gyrus, BA 22 & 77 & 3.8 & $47 /-45 / 9$ \\
\hline Right/left anterior cingulate gyrus, BA 24 & 91 & 3.8 & $6 / 27 /-2$ \\
\hline Right medial frontal gyrus, BA 6 & 175 & 3.4 & $14 / 27 / 34$ \\
\hline Left supramarginal gyrus, BA 40 & 93 & 3.0 & $-52 /-45 / 31$ \\
\hline \multicolumn{4}{|l|}{ Older adults (anodal) $<$ younger adults } \\
\hline Left/right occipital lobe, BA 18 & 1032 & -4.7 & $-14 /-93 / 9$ \\
\hline Left postcentral gyrus, BA 3 & 321 & -4.0 & $-23 /-30 / 64$ \\
\hline Left paracentral lobe, BA 6 & 104 & -3.9 & $-6 /-30 / 61$ \\
\hline Left cingulate gyrus, BA 24 & 264 & -3.8 & $-6 /-12 / 42$ \\
\hline
\end{tabular}

$B A$, Brodman area; $R$, right; $L$, left; $k$, cluster extent (number of voxels); $x / y / z$, coordinates of peak voxel in significant clusters (Talairach space); if clusters extend into both hemispheres, this is indicated by left/right (if peak voxel is located in the left hemisphere) or right/left (if peak voxel is located in the right hemisphere).

\section{Self-reported mood}

All subjects tolerated the stimulation well, and no adverse effects were reported. As in previous studies that used $1 \mathrm{~mA}$ (Gandiga et al., 2006) and our own previous study that used the same setup (Meinzer et al., 2012b), a poststudy questionnaire indicated that older participants in our study could not differentiate between atDCS and sham stimulation. No differences were found between pre/post ratings (VAMS/PANAS) for the two stimulation conditions in the older group, indicating that positive and negative effects and mood were not affected by atDCS (PANAS, mean \pm SEM; sham pre/post positive score, $3.0 \pm 0.2 / 3.0 \pm 0.2$; negative score, $1.2 \pm 0.1 / 1.1 \pm 0.1$; atDCS positive score, $2.9 \pm 0.1 / 2.8 \pm$ 0.1 ; negative score, $1.2 \pm 0.1 / 1.1 \pm 0.1$; VAMS: mean \pm SEM sham pre/post positive score, $51.1 \pm 7.3 / 44.8 \pm 8.3$; negative score, $7.1 \pm 1.2 / 6.1 \pm 1.1$; atDCS positive score, $47.4 \pm 7.5 /$ $53.3 \pm 7.2$; negative score, $10.7 \pm 2.4 / 6.5 \pm 1.2$; all $p>0.17-58$ ). The direct comparison of pre/post differences between the stimulation conditions also revealed no differences (all $p>0.26-$ $0.46)$. No significant differences in mood or effect were found between younger and older adults (all $p>0.3)$.

\section{Discussion}

The present study explored whether atDCS would improve cognitive performance during a task that is negatively affected by advanced age and simultaneously assessed atDCS-induced changes in task-related activity in elderly adults. Moreover, task-absent RS-fMRI assessed the impact of atDCS on large-scale functional RS network configuration independent of performance. Behav- iorally, atDCS improved performance in older adults up to the level of younger controls. Task-related fMRI and RS-fMRI demonstrated that a single stimulation session can temporarily reverse some age-associated changes in brain activity and connectivity.

\section{Impact of atDCS on performance}

Numerous previous studies showed beneficial effects of atDCS on cognition in younger adults (Turi et al., 2012). However, only three studies assessed atDCS effects in healthy elderly subjects and showed that atDCS improved cognitive functions in aging adults using picture naming, working memory, or object-location learning tasks (Holland et al., 2011; Berryhill and Jones, 2012; Flöel et al., 2012). These findings were confirmed and extended in the present study. In line with previous work that demonstrated improved word retrieval after IFG stimulation in younger adults (Iyer et al., 2005; Cattaneo et al., 2011; Meinzer et al., 2012b), atDCS significantly improved word retrieval in elderly adults. Moreover, by including a younger control group and using a task that is negatively affected by advanced age, we provide direct evidence that atDCS can ameliorate age-associated cognitive decline. The results of the present study and those of previous behavioral studies using different tasks (Berryhill and Jones, 2012; Flöelet al., 2012) emphasize the potential of atDCS to improve cognition in aging across different functional domains.

\section{Impact of atDCS on task-related activity}

The most consistent finding in functional imaging studies of aging is more pronounced activity in prefrontal brain areas in older versus younger adults (Goh, 2011; Grady, 2012). A compensatory functional role of this pattern has been suggested; however, there is evidence that it may not be universally beneficial (Spreng et al., 2010). Specifically, during language tasks that are strongly leftlateralized in younger adults, enhanced right frontal activity in aging can be associated with reduced performance (Crosson et al., 2013). Moreover, increased task demands during word retrieval also result in increased prefrontal activity in younger subjects, and this pattern can be observed at lower levels of task difficulty in older adults (Meinzer et al., 2012a), possibly explained by enhanced challenges posed on executive control processes caused by age-associated structural brain deterioration (Grady, 2012). In line with these interpretations, reduced performance in older adults during sham was associated with more pronounced activity in bilateral VIFG and right MFG, and enhanced right MFG activity was correlated with reduced performance.

The impact of atDCS on neural functioning during tasks that are negatively affected by aging had not been studied so far. Even in younger adults, only few studies assessed stimulation-induced brain activity changes during task-related fMRI. In these studies, atDCS resulted in decreased activity in task-relevant brain areas at the stimulation site (Meinzer et al., 2012b) or in functionally connected distant brain areas (Antal et al., 2011). Based on studies that linked reduced $\mathrm{fMRI}$ activity to superior performance or learning (Brehmer et al., 2011; Meinzer et al., 2012b), atDCSinduced activity decreases were interpreted as enhanced neural efficiency. Similarly, Holland et al. (2011) demonstrated that neural priming effects in older adults were enhanced during atDCS, expressed as more pronounced activity decreases in prefrontal cortex. In line with these studies, we showed that stimulation-induced performance improvements resulted in reduced activity in bilateral prefrontal areas that were "hyperactive" in older compared with younger adults. 
As in our previous study that used the same tDCS montage and task in younger adults (Meinzer et al., 2012b), atDCS resulted in decreased activity at the stimulation site. Given that atDCS modulates excitability in targeted regions (Stagg and Nitsche, 2011), this may reflect a direct effect of the stimulation in a specific subset of task-critical neurons. Contrary, the lefthemispheric control region (dIFG) remained unaffected by atDCS, corroborating highly specific stimulation effects in the left hemisphere in younger adults (Meinzer et al., 2012b). Moreover, enhanced activity in right-hemispheric ROIs decreased significantly during atDCS so that activity was comparable with that of younger adults. It remains to be determined whether the same physiological effects are responsible for local and remote activity decreases. This was suggested by electrophysiological studies showing that premotor cortex stimulation induced excitability changes in tightly connected primary motor cortices (Boros et al., 2008), and Antal et al. (2011) demonstrated decreased fMRI activity in the supplementary motor area during primary motor cortex stimulation. A similar effect may underlie reduced activity in the right vIFG that is functionally connected to the stimulation site (Meinzer et al., 2012b). In contrast, activity decreases in the right MFG may reflect reduced demands on top-down control processes because of more efficient left frontal processing during atDCS. Indeed, right vIFG and MFG may subserve different cognitive processes: right VIFG activity has been implicated with maintenance of information in short-term stores or inhibitory control, whereas right MFG mediates manipulation of information or monitoring of behavior (Spreng et al., 2010). Such a differential mechanism may explain why activity decreases were only correlated with behavioral improvements in the right MFG but not the bilateral vIFG.

In addition, the whole-brain analysis revealed that anterior cingulate gyrus and precuneus showed a similar pattern of enhanced (sham) and reduced (atDCS) activity as the a priori ROIs. Hyperactivity of these areas in aging is in line with previous studies (Milham et al., 2002; Persson et al., 2007; Meinzer et al., 2012c), and atDCS-induced activity reductions may reflect decreased attentional and/or monitoring demands (Carter et al., 2000; Meinzer et al., 2012c). Moreover, this whole-brain analysis also shows that atDCS did not result in unspecific task-related activity reductions across the entire brain.

\section{Impact of atDCS on resting-state $\mathrm{FMRI}$}

RS-fMRI allowed us to assess tDCS effects on large-scale functional networks in the absence of performance effects. A number of previous studies that used different resting-state data analysis approaches and addressed age-associated changes in preselected networks (Andrews-Hanna et al., 2007; Rieckmann et al., 2011; Antonenko et al., 2012) found large-scale connectivity changes in older compared with younger adults. Moreover, RS connectivity changes in aging have been linked to reduced performance (for review, see Goh, 2011). For example, enhanced coupling of bilateral prefrontal areas predicted reduced language learning ability (Antonenko et al., 2012), and disruption of connectivity between DMN components was associated with cognitive impairment in different domains (Andrews-Hanna et al., 2007; Geerligs et al., 2012). In line with these previous studies, ECM revealed widespread connectivity changes in older compared with young adults, characterized by enhanced connectivity of frontotemporal areas and reduced connectivity of sensory-motor and posterior regions. Moreover, we showed that some functional network changes in bilateral prefrontal regions overlapping with the primary language network can be reversed by atDCS. In ad- dition, ECM analysis identified atDCS-induced connectivity modulations in anterior and posterior midline areas and right temporal areas that are not part of the primary language network. Therefore, atDCS also modulated age-associated changes in other functional networks, including the DMN (Beckmann et al., 2005), known to deteriorate in healthy and pathological aging (Sperling et al., 2009; Geerligs et al., 2012). Although these largescale atDCS-induced connectivity modulations are in line with previous findings in younger adults (Keeser et al., 2011; Polaníaet al., 2011; Meinzer et al., 2012b), atDCS had differential effects on connectivity in both age groups. In younger adults, atDCS enhanced connectivity in bilateral anterior and posterior languagerelated regions (Meinzer et al., 2012b). In contrast, in older adults, atDCS reduced (hyper)connectivity in fronto-temporal language-related areas, and enhanced connectivity in sensorymotor and posterior regions. Most notably, however, although we observed both increases and decreases in connectivity during stimulation, atDCS changed the overall pattern of connectivity in the older group toward that of the younger control group.

It needs to be acknowledged that network changes identified by ECM reflect complex interactions between different networks as centrality measures take into account a given region's relationship with the entire brain (Zuo et al., 2012). Therefore, the exact mechanisms underlying the observed network modulations need to be determined in the future, and some age-associated changes were still present during atDCS; therefore, stimulation resulted only in partial reversal of age-associated connectivity changes. Moreover, whereas the present analysis shows the potential of atDCS to reduce age-associated RS network changes, future studies are required to determine the behavioral relevance of these changes.

\section{Conclusions}

In summary, our results provide converging evidence from behavioral analysis and two independent functional imaging paradigms that atDCS reduces age-associated cognitive decline and temporarily reverses age-associated alterations of functional brain activity and connectivity. Although the present study only assessed short-term stimulation effects, repeated stimulation sessions result in persistent modification of postsynaptic connections similar to long-term potentiation, which is critical for learning and neuroplasticity (Stagg and Nitsche, 2011). Therefore, our results provide a rationale to explore whether these mechanisms can be exploited to induce long-lasting cognitive improvements in normal aging or age-associated pathology. Combining repeated stimulation with cognitive training may offer an exciting new approach to delay or even reverse ageassociated cognitive decline and dementia, an issue of major importance for aging societies worldwide.

\section{References}

Andrews-Hanna JR, Snyder AZ, Vincent JL, Lustig C, Head D, Raichle ME, Buckner RL (2007) Disruption of large-scale brain systems in advanced aging. Neuron 56:924-935. CrossRef Medline

Antal A, Polania R, Schmidt-Samoa C, Dechent P, Paulus W (2011) Transcranial direct current stimulation over the primary motor cortex during fMRI. Neuroimage 55:590-596. CrossRef Medline

Antonenko D, Meinzer M, Lindenberg R, Witte AV, Flöel A (2012) Grammar learning in older adults is linked to white matter microstructure and functional connectivity. Neuroimage 62:1667-1674. CrossRef Medline

Beckmann CF, DeLuca M, Devlin JT, Smith SM (2005) Investigations into resting-state connectivity using independent component analysis. Phil Trans R Soc Lond B Biol Sci 360:1001-1013. CrossRef Medline

Berryhill ME, Jones KT (2012) tDCS selectively improves working memory 
in older adults with more education. Neurosci Lett 521:148-151. CrossRef Medline

Boros K, Poreisz C, Münchau A, Paulus W, Nitsche MA (2008) Premotor transcranial direct current stimulation (tDCS) affects primary motor excitability in humans. Eur J Neurosci 27:1292-1300. CrossRef Medline

Brehmer Y, Rieckmann A, Bellander M, Westerberg H, Fischer H, BäckmanL (2011) Neural correlates of training-related working-memory gains in old age. Neuroimage 58:1110-1120. CrossRef Medline

Carter CS, Macdonald AM, Botvinick M, Ross LL, Stenger VA, Noll D, Cohen JD (2000) Parsing executive processes: strategic vs. evaluative functions of the anterior cingulate cortex. Proc Natl Acad Sci U S A 97:1944-1948. CrossRef Medline

Cattaneo Z, Pisoni A, Papagno C (2011) Transcranial direct current stimulation over Broca's region improves phonemic and semantic fluency in healthy individuals. Neuroscience 183:64-70. CrossRef Medline

Crosson B, Garcia A, McGregor KM, Wierenga CE, Meinzer M (2013) The impact of aging on neural systems for language. In: Neuropsychology: Science and Practice (Koffler S, Morgan J, Baron IS, Greiffenstein MF, eds), pp 149-188, New York: Oxford UP.

Ferreira LK, Busatto GF (2013) Resting-state functional connectivity in normal brain aging. Neurosci Biobehav Rev 37:384-400. CrossRef Medline

Fertonani A, Rosini S, Cotelli M, Rossini PM, Miniussi C (2010) Naming facilitation induced by transcranial direct current stimulation. Behav Brain Res 208:311-318. CrossRef Medline

FlöelA, Suttorp W, Kohl O, KürtenJ, Lohmann H, Breitenstein C, Knecht S (2012) Non-invasive brain stimulation improves object-location learning in the elderly. Neurobiol Aging 33:1682-1689. CrossRef Medline

Folstein MF, Luria R (1973) Reliability, validity and clinical application of the visual analogue mood scales. Psychol Med 3:479-486. CrossRef Medline

Folstein MF, Folstein SE, McHugh PR (1975) "Mini-mental state". A practical method for grading the cognitive state of patients for the clinician. J Psychiatr Res 12:189-198. CrossRef Medline

Fox MD, Greicius M (2010) Clinical applications of resting state functional connectivity. Front Syst Neurosci 4:19. CrossRef Medline

Galluzzi S, Lanni C, Pantoni L, Filippi M, Frisoni GB (2008) White matter lesions in the elderly: pathophysiological hypothesis on the effect on brain plasticity and reserve. J Neurol Sci 273:3-9. CrossRef Medline

Gandiga PC, Hummel FC, Cohen LG (2006) Transcranial DC stimulation (tDCS): a tool for double-blind sham-controlled clinical studies in brain stimulation. Clin Neurophysiol 117:845-850. CrossRef Medline

Geerligs L, Maurits NM, Renken RJ, Lorist MM (2012) Reduced specificity of functional connectivity in the aging brain during task performance. Hum Brain Mapp. Advance online publication. Retrieved Dec. 15, 2012. doi:10.1002/hbm.22175. CrossRef Medline

Goh JO (2011) Functional dedifferentiation and altered connectivity in older adults: neural accounts of cognitive aging. Aging Dis 2:30-48. Medline

Grady C (2012) The cognitive neuroscience of ageing. Nat Rev Neurosci 13:491-505. CrossRef Medline

Holland R, Leff AP, Josephs O, Galea JM, Desikan M, Price CJ, Rothwell JC, Crinion J (2011) Speech facilitation by left inferior frontal cortex stimulation. Curr Biol 21:1403-1407. CrossRef Medline

Iyer MB, Mattu U, Grafman J, Lomarev M, Sato S, Wassermann EM (2005) Safety and cognitive effect of frontal DC brain polarization in healthy individuals. Neurology 64:872-875. CrossRef Medline

Keeser D, Meindl T, Bor J, Palm U, Pogarell O, Mulert C, Brunelin J, MöllerHJ, Reiser M, Padberg F (2011) Prefrontal transcranial direct current stimulation changes connectivity of resting-state networks during fMRI. J Neurosci 31:15284-15293. CrossRef Medline

Lindenberg R, Nachtigall L, Meinzer M, Sieg MM, FlöelA (2013) Differential effects of dual and unihemispheric motor cortex stimulation in older adults. J Neurosci 33:9176-9183. CrossRef Medline

List J, Duning T, Meinzer M, Kürten J, Schirmacher A, Deppe M, Evers S, Young P, FlöelA (2011) Enhanced rapid-onset cortical plasticity in CADASIL as a possible mechanism of preserved cognition. Cereb Cortex 21:2774-2787. CrossRef Medline

Lohmann G, Müller K, Bosch V, Mentzel H, Hessler S, Chen L, Zysset S, von Cramon DY (2001) LIPSIA-a new software system for the evaluation of functional magnetic resonance images of the human brain. IEEE Trans Med Imaging 21:449-457. Medline
Lohmann G, Margulies DS, Horstmann A, Pleger B, Lepsien J, Goldhahn D, Schloegl H, Stumvoll M, Villringer A, Turner R (2010) Eigenvector centrality mapping for analyzing connectivity patterns in FMRI data of the human brain. PloS One 5:e10232. CrossRef Medline

Lustig C, Buckner RL (2004) Preserved neural correlates of priming in old age and dementia. Neuron 42:865-875. CrossRef Medline

Meinzer M, Flaisch T, Wilser L, Eulitz C, Rockstroh B, Conway T, GonzalezRothi L, Crosson B (2009) Neural signatures of semantic and phonemic fluency in young and old adults. J Cogn Neurosci 21:2007-2018. CrossRef Medline

Meinzer M, Flaisch T, Seeds L, Harnish S, Antonenko D, Witte V, Lindenberg R, Crosson B (2012a) Same modulation but different starting points: performance modulates age differences in inferior frontal cortex activity during word-retrieval. PloS One 7:e33631. CrossRef Medline

Meinzer M, Antonenko D, Lindenberg R, Hetzer S, Ulm L, Avirame K, Flaisch T, Flöel A (2012b) Electrical brain stimulation improves cognitive performance by modulating functional connectivity and task-specific activation. J Neurosci 32:1859-1866. CrossRef Medline

Meinzer M, Seeds L, Flaisch T, Harnish S, Cohen ML, McGregor K, Conway T, Benjamin M, Crosson B (2012c) Impact of changed positive and negative task-related brain activity on word-retrieval in aging. Neurobiol Aging 33:656-669. CrossRef Medline

Milham MP, Erickson KI, Banich MT, Kramer AF, Webb A, Wszalek T, Cohen NJ (2002) Attentional control in the aging brain: insights from an fMRI study of the stroop task. Brain Cogn 49:277-296. CrossRef Medline

Nitsche MA, Paulus W (2011) Transcranial direct current stimulation-update 2011. Restor Neurol Neurosci 29:463-492. CrossRef Medline

Nitsche MA, Doemkes S, KaraköseT, Antal A, Liebetanz D, Lang N, Tergau F, Paulus W (2007) Shaping the effects of transcranial direct current stimulation of the human motor cortex. J Neurophysiol 97:3109-3117. CrossRef Medline

Oldfield RC (1971) The assessment of handedness: the Edinburgh inventory. Neuropsychologia 9:97-113. CrossRef Medline

Pantoni L, Pescini F, Nannucci S, Sarti C, Bianchi S, Dotti MT, Federico A, Inzitari D (2010) Comparison of clinical, familial, and MRI features of CADASIL and NOTCH3-negative patients. Neurology 74:57-63. CrossRef Medline

Park DC, Reuter-Lorenz P (2009) The adaptive brain: aging and neurocognitive scaffolding. Annu Rev Psychol 60:173-196. CrossRef Medline

Persson J, Lustig C, Nelson JK, Reuter-Lorenz PA (2007) Age differences in deactivation: a link to cognitive control? J Cogn Neurosci 19:1021-1032. CrossRef Medline

Polanía R, Nitsche MA, Paulus W (2011) Modulating functional connectivity patterns and topological functional organization of the human brain with transcranial direct current stimulation. Hum Brain Mapp 32:1236-1249. CrossRef Medline

Rieckmann A, Karlsson S, Fischer H, Bäckman L (2011) Caudate dopamine D1 receptor density is associated with individual differences in frontoparietal connectivity during working memory. J Neurosci 31:14284-14290. CrossRef Medline

Salvador R, Suckling J, Schwarzbauer C, Bullmore E (2005) Undirected graphs of frequency-dependent functional connectivity in whole brain networks. Philos Trans R Soc Lond B Biol Sci 360:937-946. CrossRef Medline

Sperling RA, Laviolette PS, O'Keefe K, O’Brien J, Rentz DM, Pihlajamaki M, Marshall G, Hyman BT, Selkoe DJ, Hedden T, Buckner RL, Becker JA, Johnson KA (2009) Amyloid deposition is associated with impaired default network function in older persons without dementia. Neuron 63 : 178-188. CrossRef Medline

Spreng RN, Wojtowicz M, Grady CL (2010) Reliable differences in brain activity between young and old adults: a quantitative meta-analysis across multiple cognitive domains. Neurosci Biobehav Rev 34:1178-1194. CrossRef Medline

Stagg CJ, Nitsche MA (2011) Physiological basis of transcranial direct current stimulation. Neuroscientist 17:37-53. CrossRef Medline

Thompson-Schill SL, D’Esposito M, Aguirre GK, Farah MJ (1997) Role of left inferior prefrontal cortex in retrieval of semantic knowledge: a reevaluation. Proc Natl Acad Sci U S A 94:14792-14797. CrossRef Medline

Turi Z, Paulus W, Antal A (2012) Functional neuroimaging and transcranial electrical stimulation. Clin EEG Neurosci 43:200-208. CrossRef Medline 
Watson D, Clark CA, Tellegen A (1988) Development and validation of brief measures of positive and negative affect: The PANAS scales. J Pers Soc Psychol 54:1063-1070. CrossRef Medline
Zuo XN, Ehmke R, Mennes M, Imperati D, Castellanos FX, Sporns O, Milham MP (2012) Network centrality in the human functional connectome. Cereb Cortex 22:1862-1875. CrossRef Medline 